\title{
CLINICAL DECISION SUPPORT SYSTEMS: A REVIEW ON KNOWLEDGE REPRESENTATION AND INFERENCE UNDER UNCERTAINTIES
}

\author{
GUILAN KONG, DONG-LING XU* AND JIAN-BO YANG \\ Manchester Business School (East), The University of Manchester \\ Manchester, M15 6PB, United Kingdom \\ *Email:L.Xu@mbs.ac.uk \\ Received:24-09-2007 Revised:07-04-2008
}

\begin{abstract}
This paper provides a literature review in clinical decision support systems (CDSSs) with a focus on the way knowledge bases are constructed, and how inference mechanisms and group decision making methods are used in CDSSs. Particular attention is paid to the uncertainty handling capability of the commonly used knowledge representation and inference schemes. The definition of what constitute good CDSSs and how they can be evaluated and validated are also considered. Some future research directions for handling uncertainties in CDSSs are proposed.
\end{abstract}

Keywords: clinical decision support system, inference mechanism, knowledge representation scheme, knowledge acquisition tool, group clinical decision making, uncertainty handling.

\section{Introduction}

CDSSs have almost 40 years of history. From the first generation of CDSSs such as $\mathrm{MYCIN}^{1}$ and $\mathrm{QMR}^{2}$, to the second generation such as Protégé ${ }^{3}$, and to very recent CDSSs such as a DSS for lower back pain diagnosis $^{4}$, significant research progress, both theoretically and practically, has been made since the idea of computer-based CDSSs first emerged. However several barriers continue to impede the effective implementation of CDSSs in clinical settings, among which representation of and reasoning about medical knowledge particularly under uncertainty are areas that require refined methodologies and techniques ${ }^{4,5}$.

Uncertainty exists in almost every stage of a clinical decision making process. Sources of uncertainties may include that patients can not describe exactly what has happened to them or how they feel, doctors and nurses can not tell exactly what they observe, laboratories report results may be with some degrees of error, physiologists do not precisely understand how the human body works, medical researchers can not precisely characterize how diseases alter the normal functioning of the body, pharmacologists do not fully understand the mechanisms accounting for the effectiveness of drugs, and no one can precisely determine one's prognosis ${ }^{6}$. One of the main challenges in representation of and reasoning about medical knowledge is how to rationally handle those uncertainties so that a CDSS can support clinicians to make correct and reliable diagnosis and treatment decisions. Some identified issues include the representation of associated uncertainty in clinical domain knowledge, reasoning under uncertainty, the support of a non-exclusive multi-part diagnosis and systematic clinical evaluation ${ }^{4}$.

The main purpose of the review is to identify what have been achieved in dealing with those issues in the past and to search for new directions to advance the uncertainty handling capability of CDSSs. The databases used for searching related literature in CDSSs for this review include: SicenceDirect, ISI Web of Knowledge, IEEE Xplore and Google, and the time period of the reviewed literature ranges from 1970s to date.

The paper is organised as follows. Section 2 outlines typical definitions of CDSSs together with a general CDSS model in the literature. Section 3 reviews the state of the art of CDSSs, including knowledge base construction, inference mechanisms, group clinical decision support systems (GCDSSs) and CDSSs evaluation. Finally, Section 4 concludes the review and proposes some future directions of CDSSs research. 


\section{What are CDSSs}

This section briefly describes some typical definitions of CDSSs and a general model of CDSSs which most researchers adopt in the literature of CDSSs.

\subsection{Definition of CDSSs}

In the literature, many researchers have given their definitions of CDSSs. Some typical definitions are given below. Musen ${ }^{7}$ defined a CDSS as any piece of software that takes information about a clinical situation as inputs and that produces inferences as outputs that can assist practitioners in their decision making and that would be judged as "intelligent" by the program's users. Miller and Geissbuhler ${ }^{8}$ defined a CDSS providing diagnostic decision support as a computer-based algorithm that assists a clinician with one or more component steps of the diagnostic process. Sim et al. ${ }^{9}$ defined CDSSs as "software that designed to be a direct aid to clinical decision-making, in which the characteristics of an individual patient are matched to a computerized clinical knowledge base and patientspecific assessments or recommendations are then presented to the clinician or the patient for a decision".

In more recent studies, researchers have been trying to classify CDSSs in the literature so as to provide a holistic picture of CDSSs. For example, Berlin et al. ${ }^{10}$ did research on a CDSS taxonomy to describe the technical, workflow, and contextual characteristics of CDSSs, and the research results are very useful for researchers to have a comprehensive understanding of various designs and functions of CDSSs.

\subsection{A General Model of CDSSs}

A general model of $\mathrm{CDSSs}^{11,12}$ which has been discussed in the literature is shown as Figure 2.1.

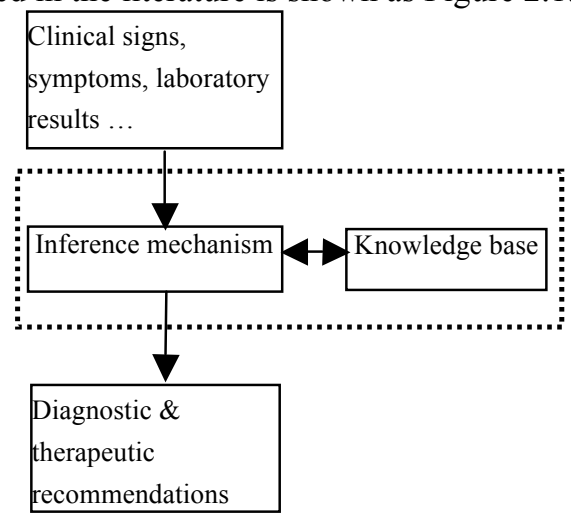

Figure 2.1: The General Model of CDSSs
As seen from Figure 2.1, there are inputs composed of clinical signs, symptoms, laboratory tests and so on to the system and outputs including diagnostic and therapeutic recommendations from the system. The system has two basic architectural components: a knowledge base and an inference (reasoning) mechanism. The knowledge base is a structured collection of expert medical knowledge used by the CDSS. The inference mechanism is a set of computer algorithms used to process clinical signs, symptoms and laboratory test results in relation to the knowledge base.

According to the general CDSS model described in Figure 2.1, the system users interact with the CDSS in an iterative fashion, selectively entering clinical signs, symptoms and laboratory test results, and using the CDSS output recommendations to assist with the diagnostic and therapeutic decision-making processes.

\section{The Current Status of CDSSs}

This section reviews current CDSSs from different perspectives. Firstly, in Sections 3.1 and 3.2, based on the general CDSS model described in Section 2.2, knowledge base construction methods, including knowledge representation schemes and knowledge acquisition tools, and inference mechanisms used in existent CDSSs are considered for review. Then Section 3.3 reviews GCDSSs. Finally Section 3.4 is devoted to reviewing current CDSS evaluation practices.

\subsection{Knowledge Base Construction}

As to what makes a good clinical decision support system, Purcell ${ }^{13}$ held the view that a CDSS is only as effective as its underlying knowledge base. From the analysis and design of a CDSS at the very beginning to the implementation of the CDSS at the final stage, the way knowledge base is being exploited for clinical decision support is one of the most key facets of a successful CDSS. This section focuses on the review of this important facet, including knowledge representation schemes and knowledge acquisition tools.

\subsubsection{Knowledge representation schemes}

Carter $^{14}$ argued that the goal of knowledge representation is to provide intelligent systems with information about a specific domain in a form that can be processed efficiently. Also he classifies knowledge 
representation schemes into four categories: logic, procedural, graph/network, and structured. This paper will review knowledge representation schemes according to these four categories.

Firstly, from the literature, logic seems to be the most common representation format used by researchers in the field of general artificial intelligence. In general, medical knowledge can be divided into two types: declarative knowledge and procedural knowledge. Declarative knowledge includes propositions and sentences. Propositions are statements about the world that are either "true" or "false". These statements may be connected by boolean operators such as "and", "or", and "and not" to form sentences. Procedural knowledge provides more explicit information about what action can be taken or what conclusion can be drawn from declarative knowledge. For example, "'ElectroCardioGram (ECG) shows $\geq 2 \mathrm{~mm}$ ST elevation in two contiguous chest leads' or 'ECG shows $\geq 1 \mathrm{~mm}$ ST elevation in two contiguous limb leads" is declarative knowledge, and "IF 'ECG shows $\geq 1 \mathrm{~mm}$ ST elevation in two contiguous limb leads', THEN 'there is a strong evidence of ST-Segment Elevation Myocardial Infarction (STEMI)" " is procedural knowledge. The logic-based representations are declarative in nature, in that they consist of "true" or "false" statements and all questions are resolved through standard logic inference mechanism which is simply a "look up" of known facts $^{14}$.

Secondly, procedural knowledge representation, on the other hand, is not simply a "look up" of known facts. It offers a "process" to aid diagnostic and therapeutic decision-making ${ }^{14}$. Procedural knowledge in medicine is usually provided in the form of rules in existent CDSSs. Many implemented CDSSs, from the very early CDSSs such as MYCIN, PUFF ${ }^{15}$, and IMM/Serve ${ }^{16}$, to recently developed CDSSs such as unified medical language system (UMLS)-based $\mathrm{CDSS}^{17}$ and Chinese medical diagnostic system $(\mathrm{CMDS})^{18}$ are all rule-based. Actually, rules have been the dominant knowledge representation scheme for medical expert systems since the days of $\mathrm{MYCIN}^{14}$.

In practice, because of the existence of uncertainty in medical domain knowledge, clinical signs and symptoms, some CDSSs use logic, rules as well as probabilities to represent knowledge with uncertainties. Fuzzy logic ${ }^{19,20}$ and Bayes' rule ${ }^{21}$ are used by researchers in designing knowledge representation schemes. Bayes' rule has a limitation that it is based on the simplified assumption that most clinical signs and symptoms are independent of one another ${ }^{22}$.

Thirdly, networks including Bayesian belief network $^{23,24}$, decision trees $^{25,26}$ and artificial neural networks ${ }^{27}$ are used in CDSSs.

In CDSSs design, the choice of adopting a Bayesian network as representation scheme allows one to explicitly take advantage of conditional independencies from the modelling viewpoint, and to rely on several powerful algorithms for probabilistic inference ${ }^{28}$. Decision trees are frequently used in guideline-based CDSSs targeted for therapeutic recommendations, such as $\mathrm{EsPeR}^{29}$ system. The advantage of decision trees is that they are simple to understand and interpret, but they are always used together with other representation schemes. Types of artificial neural networks used in CDSSs include feedforward neural network, recurrent network, stochastic neural network, and modular neural network. The greatest advantage of artificial neural networks is that they have the ability to "learn" from the observed data. The disadvantage is that they are unable to provide reliable and logical representation of knowledge beyond their learnt zones.

Fourthly, structural representations emphasize the "packaging" of knowledge into well defined pieces with higher levels of organizations ${ }^{14}$. "Frame" was the first widely accepted structural knowledge representation format created by Minsky ${ }^{30}$. Some CDSSs such as earlier CENTAUR ${ }^{31}$ and Arden Syntax ${ }^{32}$ all use frame as one of their representation formats.

In recent CDSS studies, database management systems (DBMS) are frequently used to store and manage structural knowledge. Most CDSSs use relational database to record patient history data and clinical signs and symptoms. Some CDSSs use objectoriented database management systems (OODBMS) to store medical knowledge, which are limited by data types in relational databases ${ }^{33}$. DBMS is good at storing declarative and procedural medical knowledge with or without uncertainty. However, DBMS has a major drawback. Although its structured query language (SQL) can manipulate "query", "add", "update" and "delete" to its stored objects, it lacks a specific knowledge inference mechanism to reason and draw logic conclusions from the data.

Apart from the four knowledge representation schemes discussed above, there are special knowledge 
representation schemes developed to represent temporal and spatial medical knowledge $\mathrm{3}^{34,35}$.

As analyzed above, most knowledge representation schemes have their own advantages and drawbacks. The choice of an appropriate knowledge representation scheme in the construction of a knowledge base depends on the domain knowledge it represents and the inference mechanism it uses.

\subsubsection{Knowledge acquisition tools}

Knowledge acquisition is a very important starting procedure for the construction of knowledge bases in CDSSs. The first step of knowledge acquisition is to select the target clinical area and select expert clinicians to gain domain specific knowledge. The next step is then to transfer the knowledge into computer interpretable knowledge based on the designed knowledge representation schemes. This section is focused on the review of the knowledge acquisition tools, the purpose of which is to employ ready-made templates to acquire clinical domain knowledge from experts.

Many knowledge acquisition tools have been developed for CDSSs. Among them, some tools ${ }^{17,18}$ are designed for acquiring medical domain specific knowledge, and others ${ }^{36,37,38,39}$ are designed specially for the acquisition of clinical guidelines which can be used as the best and standardized clinical procedures.

In Table 3.1, the comparisons of some acquisition tools are summarized from the following 5 facets: Established domain ontology, Abilities of handling uncertainties, Representation schemes, Specific knowledge representation language and Guideline execution engine or inference engine.

Some other guideline-based CDSSs which are frequently mentioned in the literature such as $\mathrm{GLIF}^{40}$, $E^{4} N^{41}$ and Arden Syntax are not explicitly listed in Table 3.1, but they are represented by Protégé and UMLS-based knowledge acquisition tool in the table. GLIF and EON use Protégé knowledge acquisition tools. Arden Syntax focuses on a representational syntax and format for sharing modular medical knowledge, and its main contribution is the representation format of MLM that is used in UMLS-based knowledge acquisition tool.
As the handling of uncertainty is one of the major challenges for most knowledge acquisition tools, the following review is mainly focused on the comparison of the "abilities of handling uncertainties".

In Table 3.1, PROforma ${ }^{38}$ is a guideline acquisition tool that contains expressive constructs for describing uncertain aspects of a guideline ${ }^{42}$. Its ability to make decision under uncertainty is provided by means of argumentation mechanism. In this kind of argumentation mechanism, diagnostic and therapeutic decisions are defined in terms of a set of options, and the decisions are made by using argument rules and commitment rules. Argument rules can support or oppose a decision option, and eventually establish a preference order on the options. Commitment rules are used for the selection of a decision option based on the preference order that argument rules have established. However, if a decision can be made without uncertainty, then those argument rules can be ignored and the decision can be made solely by using the commitment rules $^{38}$. GLARE ${ }^{39}$ has restricted capability in handling uncertainties in diagnostic decisions. In GLARE, diagnostic decisions are represented as a set of triples: $<$ diagnosis, parameter, score $>$ (where, in turn, a parameter is a triple of $<$ data, attribute, value $>$ ), a threshold value is used to compare each diagnosis' score, and all alternative diagnoses are shown to the user - a physician, together with their scores and the threshold value. GLARE lets the user to choose diagnostic decisions among those alternatives that the system provides in a list. A warning is given if the user chooses a diagnosis whose score does not exceed the threshold value. AsbruView ${ }^{36}$ can handle uncertainty in temporal scopes by "time annotations" which is used to specify the temporal aspects of its structured representation format: time-oriented, skeletal plans. A time annotation specifies different points in time and duration in relation to a reference point in time, such as the earliest starting shift (ESS), the latest starting shift (LSS), the earliest finishing shift (EFS), the latest finishing shift (LFS), the minimal duration (MinDu) and the maximal duration (MaxDu). These data specify the temporal constraints within which an action must be taken or a condition must be satisfied in order to trigger an action. The time annotation allows a representation of uncertainty in starting time, ending time, and duration of a plan. The UMLS-based knowledge acquisition tool 
Clinical Decision Support Systems: A Review on Knowledge Representation and Inference under Uncertainties 5

does not take uncertainties into consideration during its design and development processes. The CMDS: objectoriented knowledge acquisition editor has a better function of handling uncertainty, because it associates with each rule a certainty factor that represents how true the rule is. The certainty factor ranges from -1 to 1 , where -1 means the rule is known to be false, 0 means no information is known, and 1 means the rule is known to be true. For example, after the user defines a rule called "Rule1": "IF the symptom pattern is S, THEN the disease is D", the system would pop out a dialogue box asking the user to input rule settings about "Rule1", and the settings include the certainty factor of the rule. Protégé deals little with uncertainty.

Table 3.1 Knowledge Acquisition Tools Comparisons

\begin{tabular}{|c|c|c|c|c|c|}
\hline Knowledge & $\begin{array}{l}\text { Established } \\
\text { domain ontology }\end{array}$ & $\begin{array}{l}\text { Abilities of handling } \\
\text { uncertainties }\end{array}$ & $\begin{array}{l}\text { Representation } \\
\text { schemes }\end{array}$ & $\begin{array}{l}\text { Supported by a } \\
\text { specific knowledge } \\
\text { representation language }\end{array}$ & $\begin{array}{l}\text { Need a guideline } \\
\text { execution engine } \\
\text { /inference engine }\end{array}$ \\
\hline PROforma & $\mathrm{N} / \mathrm{A}$ & $\begin{array}{l}\text { Use argumentation } \\
\text { mechanism to make } \\
\text { diagnostic and therapeutic } \\
\text { decisions }\end{array}$ & $\begin{array}{l}\text { Structured } \\
\text { (Object-oriented } \\
\text { representation: } \\
\text { Plans) and rules }\end{array}$ & Yes & $\begin{array}{l}\text { Yes } \\
\text { (PROforma } \\
\text { enactment engine) }\end{array}$ \\
\hline GLARE & Yes & $\begin{array}{l}\text { Use a threshold value to } \\
\text { compare the diagnosis' score }\end{array}$ & $\begin{array}{l}\text { Structured } \\
\text { (Object-oriented } \\
\text { representation: } \\
\text { Actions) }\end{array}$ & Yes & Yes \\
\hline Protégé & Yes & Not mentioned & $\begin{array}{l}\text { Structured } \\
\text { (Frames) }\end{array}$ & Yes & Yes \\
\hline AsbruView & $\mathrm{N} / \mathrm{A}$ & $\begin{array}{l}\text { Use "time annotations" to } \\
\text { represent uncertainty in } \\
\text { starting time, ending time, } \\
\text { and duration of a plan }\end{array}$ & $\begin{array}{l}\text { Structured } \\
\text { (Time-Oriented, } \\
\text { Skeletal Plans) }\end{array}$ & Yes & Yes \\
\hline $\begin{array}{l}\text { UMLS-based } \\
\text { knowledge } \\
\text { acquisition tool }\end{array}$ & Yes & Not mentioned & $\begin{array}{l}\text { Logic and rules } \\
\text { ( Medical Logic } \\
\text { Modules (MLMs) }\end{array}$ & Not mentioned & Yes \\
\hline $\begin{array}{l}\text { CMDS: object- } \\
\text { oriented knowledge } \\
\text { acquisition editor }\end{array}$ & Yes & $\begin{array}{l}\text { Use certainty factor or belief } \\
\text { degree to model uncertainty }\end{array}$ & Rules & Not mentioned & Yes \\
\hline
\end{tabular}

Except for PROforma and AsbruView, those medical domain specific knowledge or clinical guideline acquisition tools listed in Table 3.1 have a common characteristic: the established domain ontology, such as a set of ready-made and configurable templates. Although the ontology provides guided assistance for expert physicians to express their medical knowledge into a computer-interpretable format, it may also restrict some of their knowledge from being extracted. This is because in reality CDSS developers may only have limited medical knowledge in a chosen domain and the developed ontology may not be able to cover all the formats required to express the domain knowledge. For example, the UMLS-based knowledge acquisition tool allows medical experts to configure ready made rulebased templates which are derived from the UMLS, but the UMLS only covers a restricted number of clinical domains. Another shortcoming of the ready made rule templates in the UMLS-based knowledge acquisition tool is that it has restricted capability in handling uncertainties and conflict in rules.

\subsection{Inference Mechanisms Used in Existent CDSSs}

After reviewing the current status of knowledge base construction in Section 3.1, this section focuses on inference mechanisms used in existent CDSSs.

From the literature, inference mechanisms used in CDSSs include rule based, Bayesian, Bayesian belief networks, heuristic, semantic network, neural networks, genetic algorithms and case-based.

In rule-based CDSSs, sets of boolean "if-then" rules are processed. The forward and backward chaining of rules may be used to conclude a diagnosis and provide diagnostic explanations for clinical users ${ }^{43}$. 
Bayesian systems predict the posterior probability of diagnoses based on the prior disease probabilities, and the sensitivity and specificity of confirmed clinical signs and symptoms ${ }^{44}$.

Bayesian belief networks are often created as reformulations of traditional Bayesian representations and can provide many of the same browsing and explanation capabilities of traditional systems ${ }^{45}$.

Heuristic systems include statistical measures. Some statistical methods such as support vector machine $(\mathrm{SVM})^{46}$ and least square support vector machine (LSSVM $)^{47}$ have been proposed for medical reasoning.

In regard to semantic network, since most medical knowledge is ill-structured and involves uncertainties, it is difficult to use a pure semantic network to make clinical inference in CDSSs.

Neural networks are frequently used by researchers as inference mechanism because during the development of this kind of CDSSs, developers are not required to understand the relationship between input and output variables. Neural networks are a black box modelling technique that models relationships by learning from historical data, while developers of CDSSs based on Bayesian networks need to have sufficient domain knowledge including related probabilities. Li et al. ${ }^{48}$ compared neural networks with other mathematical models for building a traumatic brain injury (TBI) medical decision support system (MDSS) in their study, and the results suggest that neural networks may be a better solution for complex, non-linear CDSS than conventional statistical techniques. The disadvantage of a neural network is that the "rules" that the network uses do not follow a particular logic and are not explicitly understandable.

Genetic algorithms ${ }^{49,50}$ have an advantage that by iteratively extracting the best solutions, an optimal solution which is the fittest can be reached, but how to define the fitness is a challenge in genetic algorithms ${ }^{22}$.

In recent studies, some inference methodologies are combined together to provide medical reasoning, such as LSSVM with fuzzy weighting ${ }^{47}$, and artificial neural networks with fuzzy weighting ${ }^{51}$.

When medical knowledge is difficult to be modelled in the format of logical representation, medical experts will turn to concrete examples to express their knowledge. In this situation, the case-based reasoning (CBR) approach is used in CDSSs. The advantage of
CBR is that concrete similar empirical clinical cases are more convincing than some other implicit medical knowledge. The disadvantages of CBR include that it is difficult to measure the similarity between cases, the retrieval process is hard to be accurate and efficient, and the input scheme required by the CDSS based on CBR may not be easily accepted by clinicians.

\subsection{GCDSSs Status}

Group clinical decision making is another important research area of CDSSs. However, in the literature, there are currently not many publications on it yet. In early 1990s, Rao et al. ${ }^{52,53}$ found that although group decision making is wide spread in medicine, limitations in technology and other factors limited the growth of GCDSSs for medical decision making (MDM). In 1996, Rao et al. ${ }^{54}$ published an analysis on the classification of MDM from a GCDSS perspective. Later, in 2000, Rao and Turoff proposed a hypermedia-based GCDSS to support collaborative $\mathrm{MDM}^{55}$, MEDICALWARE ${ }^{\mathrm{TM}}$, which, integrated with a GCDSS, is designed to provide problem-solving support, access to clinical algorithms and procedures, expert inference support and several MDM support tools with hypermedia functionality.

\subsection{CDSSs Evaluation Status}

Evaluation is a crucial component in the development of any $\mathrm{CDSS}^{56}$. In the literature, Miller ${ }^{16}$ argued that formal evaluation of CDSSs should take into account the following four perspectives:

(1) appropriate evaluation design;

(2) specification of criteria for determining CDSSs efficacy in the evaluation;

(3) evaluation of the boundaries or limitations of CDSSs;

(4) identification of potential reasons for "lack of system effect".

Keith and Greene ${ }^{57}$ adopted the following examinations:

(1) evaluation of the expert knowledge;

(2) evaluation of the integrated system;

(3) external validation of the system;

(4) in-house online trial;

(5) multicenter randomized trial in evaluation of their system.

Thomas et al. ${ }^{58}$ used case scenarios to evaluate their guideline-based CDSS. Becker et al. ${ }^{59}$ evaluated their 
CDSS by validating not only the knowledge base, but also the inference mechanism.

Most published papers on CDSSs evaluation have focused on the issue of system accuracy, with few studies evaluating the impact of using a CDSS on clinical care ${ }^{56}$

\section{Conclusions}

After a critical review of the literature on CDSSs, a conclusion can be drawn that a number of knowledge bases and inference mechanisms in CDSSs have been developed, many of which show promise for making a significant impact on patient care. However, after decades of the development of these programs, no CDSS is widely used by physicians ${ }^{14}$.

Miller and Geissbuhler ${ }^{8}$ argued that there are a number of problems that have limited the ultimate success of CDSSs to date. These include difficulties with domain selection and knowledge base construction and maintenance, problems with the diagnostic algorithms and user interfaces, and problems with system evaluation.

In recent studies, Kawamoto et al. ${ }^{60}$ tried to identify four features of CDSSs as independent predictors of a good CDSS: automatic provision of decision support as part of clinician workflow, provision of recommendations rather than just assessments, provision of decision support at the time and location of decision making, and computer based decision support.

To achieve those four features identified by Kawamoto et al., a CDSS should have a clinical domain knowledge base which has been validated in practice, an intelligent diagnostic inference mechanism which can handle medical uncertainties, and accurate diagnostic or therapeutic recommendations and a friendly user interface that can be easily accepted and used by clinicians.

With the rapid development of networking and database technologies, the problem of developing an adequate database which can store both declarative and procedural knowledge may not be difficult to overcome. However, it is not easy to model uncertain clinical domain knowledge and structure the knowledge base so that the knowledge can be easily accessed, expanded, updated and maintained. Unable to reason with uncertain knowledge to provide informative clinical decision support is still one of the weaknesses in most, if not all, implemented CDSSs
Since uncertainty is unavoidable in medical reasoning ${ }^{6}$, it is critical that future research in CDSSs should address the problems in knowledge representation and inference under uncertainties. One potential way of overcoming the difficulties and weaknesses is to explore the potential offered by the latest development in decision sciences, especially the branch of decision making under uncertainty. For example, the recently developed belief rule-base inference methodology using the evidential reasoning approach $(\text { RIMER) })^{61}$ may be used for clinical knowledge representation and inference under uncertainties. In RIMER, a rule base is designed with belief degrees embedded in all possible consequents of a rule. Such a rule base is capable of capturing vagueness, incompleteness, and nonlinear causal relationships, while traditional if-then rules can be represented as a special case. Inference in such a rule base is implemented using the evidential reasoning (ER) approach $^{62-64}$.

To conclude, some possible future research directions in CDSSs may include:

(1) a more informative knowledge representation scheme which can represent clinical domain knowledge accurately;

(2) a more reliable inference mechanism which can reason with information having different types and degrees of uncertainties;

(3) intelligent learning capability to automatically update reasoning rules when the diagnoses and recommendations provided by the CDSS are overwritten by an authorized clinician;

(4) support of group or collaborative clinical decisionmaking.

\section{References}

1. E. H. Shortliffe, Computer-Based Metrical Consultations: MYCIN (Elsevier, New York, 1976).

2. R. A. Miller, F. E. J. Masarie, Methods of Information in Medicine 28, 340 (1989).

3. M. A. Musen, J. H. Gennari, H. Eriksson, S. Tu, A. R. Puerta, Medinfo 8, 766 (1995).

4. L. Lin, P. J.-H. Hu, O. R. Liu Sheng, Decision Support Systems 42, 1152 (2006).

5. M. Musen, Y. Shahar, E. Shortliffe, in Biomedical Informatics. (2006) pp. 698-736.

6. P. Szolovits, Methods of Information in Medicine 34, 111 (1995).

7. M. A. Musen, in Handbook of medical informatics $\mathrm{J}$. H. V. a. M. Bemmel, M. A. , Ed. (Bohn Stafleu Van Loghum, Houten, 1997). 
8. R. A. Miller, A. Geissbuhler, in Clinical Decision Support Systems E. S. Berner, Ed. (Springer-Verlag, New York, 1999), vol. 3-34.

9. I. Sim et al., J Am Med Inform Assoc 8, 527 (November 1, 2001, 2001).

10. A. Berlin, M. Sorani, I. Sim, Journal of Biomedical Informatics 39, 656 (2006).

11. J. Reggia, Annals of biomedical engineering 9, 605 (1981).

12. M. J. Lincoln, in Clinical Decision Support Systems E. S. Berner, Ed. (Springer-Verlag, New York, 1999) pp. 105-138.

13. G. P. Purcell, BMJ 330, 740 (April 2, 2005, 2005).

14. J. H. Carter, in Clinical Decision Support Systems E. S. Berner, Ed. (Springer-Verlag, New York, 1999) pp. 169-198.

15. J. S. Aikins, J. C. Kunz, E. H. Shortliffe, R. J. Fallat, Computers and Biomedical Research 16, 199 (1983).

16. P. Miller et al., in Proceedings : a conference of the American Medical Informatics Association / ... AMIA Annual Fall Symposium. (Hanley And Belfus, 1996) pp. 208-212.

17. S. L. Achour, M. Dojat, C. Rieux, P. Bierling, E. Lepage, J Am Med Inform Assoc 8, 351 (July 1, 2001, 2001).

18. M.-J. Huang, M.-Y. Chen, Expert Systems with Applications 32, 658 (2007).

19. S. Shiomi et al., J Nucl Med 36, 593 (April 1, 1995, 1995).

20. S. Suryanarayanan, N. P. Reddy, E. P. Canilang, International Journal of Bio-Medical Computing 38, 207 (1995).

21. H. R. J. Warner, Methods of Information in Medicine 28, 370 (Nov, 1989).

22. S. A. Spooner, in Clinical Decision Support Systems E. S. Berner, Ed. (Springer-Verlag, New York, 1999) pp. 35-60.

23. R. Montironi, P. H. Bartels, D. Thompson, M. Scarpelli, P. W. Hamilton, Analytical and quantitative cytology and histology 16, 101 (1994).

24. P. H. Bartels, D. Thompson, M. Bibbo, J. Weber, Analytical and quantitative cytology and histology 14, 459 (Dec, 1992).

25. P. Kokol, M. Mernik, J. Završnik, K. Kancler, M. Ivan, Journal of Medical Systems 18, 201 (August, 1994).

26. V. Podforelec, P. Kokol, in Engineering in Medicine and Biology Society, 1998. Proceedings of the 20th Annual International Conference of the IEEE. (Hong Kong, 1998), vol. 3, pp. 1202-1205 vol.3.

27. W. Baxt, Annals of internal medicine 115, 843 (Dec 1, 1991).

28. P. T. Stefania Montani, International Journal of Intelligent Systems 21, 585 (2006).

29. I. Colombet et al., International Journal of Medical Informatics 74, 597 (2005).

30. M. Minsky, in Mind Design J. Haugeland, Ed. (MIT Press, Cambridge, MA, 1981) pp. 95-128.
31. J. S. Aikins, Doctoral thesis (1980).

32. P. D. Clayton, T. A. Pryor, O. B. Wigertz, G. Hripcsak, in Thirteenth Annual Symposium on Computer Applications in Medical Care: Proceedings L. C. Kingsland, Ed. (IEEE Computer Society Press New York, 1989) pp. 116-121.

33. F. Pinciroli, C. Combi, G. Pozzi, Medical informatics 17, 231 (Oct-Dec, 1992).

34. J. F. Allen, Artificial Intelligence 23, 123 (1984).

35. Y. Shahar, M. A. Musen, Computers and Biomedical Research 26, 255 (Jun, 1993).

36. S. Miksch, R. Kosara, Y. Shahar, P. Johnson, in Proceedings of the 4th International Conference on Artificial Intelligence Planning Systems 1998 (AAAI Press, Menlo Park, CA, 1998) pp. 11-18.

37. R. A. Greenes, A. Boxwala, W. Sloan, L. OhnoMachado, S. R. Deibel, in AMIA 1999 Annual Symposium Proceedings M. L. Nancy, Ed. (American Medical Informatics Association, Washington, DC, 1999) pp. 261-265.

38. J. Fox, N. Johns, A. Rahmanzadeh, Artificial Intelligence in Medicine 14, 157 (1998).

39. P. Terenziani, G. Molino, M. Torchio, Artificial Intelligence in Medicine 23, 249 (2001).

40. L. Ohno-Machado et al., J Am Med Inform Assoc 5, 357 (July 1, 1998, 1998).

41. M. A. Musen, S. W. Tu, A. K. Das, Y. Shahar, J Am Med Inform Assoc 3, 367 (November 1, 1996, 1996).

42. P. A. de Clercq, J. A. Blom, H. H. M. Korsten, A. Hasman, Artificial Intelligence in Medicine 31, 1 (2004).

43. E. H. Shortliffe, L. E. Perreault, Medical Informatics (Addison Wesley Publishing, Reading, MA, 1990).

44. H. R. Warner, Computer-Assisted Medical Decision Making (Academic Press, New York, 1979).

45. Y. C. Li, P. J. Haug, H. R. Warner, in Proceedings / the ... Annual Symposium on Computer Application [sic] in Medical Care. (American Medical Informatics Association, 1994) pp. 765-769.

46. L. Guo, W. Yan, Y. Li, Y. Wu, X. Shen, paper presented at the Annual International Conference of the IEEE Engineering in Medicine and Biology Society. IEEE Engineering in Medicine and Biology Society 2005.

47. E. Comak, K. Polat, S. Gunes, A. Arslan, Expert Systems with Applications 32, 409 (2007).

48. Y.-C. Li, L. Liu, W.-T. Chiu, W.-S. Jian, International Journal of Medical Informatics 57, 1 (2000).

49. J. W. Grzymala-Busse, L. K. Woolery, in Proceedings / the ... Annual Symposium on Computer Application [sic] in Medical Care. (American Medical Informatics Association, 1994) pp. 730-734.

50. M. Levin, M.D.Computing: computers in medical practice 12, 193 (May-Jun, 1995).

51. K. Polat, S. Gunes, Digital Signal Processing 16, 913 (2006). 
52. G. R. Rao, B. A. Suresh, M. Turoff, S. R. Hiltz, in Proceedings of the 16th Annual International Conference of the IEEE. (1994), vol. 2, pp. 13541355

53. G. R. Rao, B. A. Suresh, in Proceedings of the First Regional Conference., IEEE. (1995) pp. 1/81-1/82.

54. G. R. Rao, B. A. Suresh, M. Turoff, in Proceedings of the 18th Annual International Conference of the IEEE. (1996), vol. 5, pp. 2205-2206

55. G. R. Rao, M. Turoff, Decision Support Systems 30, $187(2000)$.

56. E. S. Berner, in Clinical Decision Support Systems E. S. Berner, Ed. (Springer-Verlag, New York, 1999) pp. $169-198$.

57. R. D. F. Keith, K. R. Greene, Bailliere's Clinical Obstetrics and Gynaecology 8, 583 (1994).

58. K. W. Thomas, C. S. Dayton, M. W. Peterson, Journal of Medical Internet Research 1, e6 (Nov 19, 1999).

59. K. Becker et al., Artificial Intelligence in Medicine 11, 33 (1997).

60. K. Kawamoto, C. A. Houlihan, E. A. Balas, D. F. Lobach, BMJ 330, 765 (April 2, 2005, 2005).

61. J. B. Yang, J. Liu, J. Wang, H. S. Sii, H. W. Wang, Ieee Transactions on Systems Man and Cybernetics Part a-Systems and Humans 36, 266 (Mar, 2006).

62. J.-B. Yang, P. Sen, Systems, Man and Cybernetics, IEEE Transactions on 24, 1458 (1994).

63. J.-B. Yang, M. G. Singh, Systems, Man and Cybernetics, IEEE Transactions on 24, 1 (1994).

64. J. B. Yang, D. L. Xu, Ieee Transactions on Systems Man and Cybernetics Part a-Systems and Humans 32, 289 (May, 2002). 